\title{
Beinbrech oder Moorlilie (Narthecium ossifragum)
}

\author{
Kurt Baumann
}

\begin{abstract}
Bog Asphodel (Narthecium ossifragum, Nartheciaceae) is flower of the year 2011. The derivation of its name, biology and ecology are explained. It is not clear whether or not Bog Asphodel is a poisonous plant, perhaps it is toxic for sheep.

\section{Zusammenfassung}

Beinbrech (Narthecium ossifragum, Nartheciaceae) ist Blume des Jahres 2011. Namensherkunft, Biologie und Ökologie werden erläutert. Es gibt verschiedene Angaben zur Giftigkeit des Beinbreches. Möglicherweise ist er für Schafe toxisch.
\end{abstract}

\section{Einführung}

Der Beinbrech wurde von der Stiftung Naturschutz Hamburg und der Stiftung LoKI Schмidt zum Schutze gefährdeter Pflanzen zur Blume des Jahres 2011 gewählt. Mit ihrer Wahl soll auf die Gefährdung des Lebensraumes Moor hingewiesen werden. Es war die letzte (die 32.) Wahl einer Blume des Jahres, an der die im Herbst 2010 verstorbene LoKi SchMidT noch beteiligt war. Wie schon früher entschied man sich für eine Pflanze aus dem norddeutschen Raum.

\section{Verwandtschaft, Arten und Namensherkunft}

Aufgrund des Blütenbaus wurde Beinbrech früher zu den Liliengewächsen gezählt und noch Haeupler \& Muer (2007) gruppierten sie bei den Liliaceae im engeren Sinne ein. Die Liliaceae werden nun in drei Unterfamilien aufgeteilt, wobei die Gattung Narthecium zunächst zu den Germeratigen (Melanthioideae) gestellt wurde, zu denen auch Germer (Veratrum) und Torflilie (Tofieldia) gehören. Von manchen Autoren (z. B. Brummit I992, Aichele 1996) wird die Unterfamilie als eigene Familie betrachtet.

Die Versuche zur Klärung verwandtschaftlicher Beziehungen beruhten früher auf morphologischen und embryologischen Merkmalen. Heute werden vor allem auch molekularbiologische Untersuchungen mit einbezogen. Aktuell wird Narthecium mit drei anderen außereuropäischen Gattungen in die eigene Familie Nartheciaceae gestellt. Sie gehört zur Ordnung der Dioscoreales, deren bekannteste Vertreter die in Süddeutschland und vor allem im Mittelmeergebiet vorkommende Schmerwurz (Tamus communis) und die tropische Yamswurzel (Dioscorea batatas) sind.

Schumacher (1945) und Tamura (1998) nennen acht Arten, darunter drei europäische: $N$. asiaticum in Japan, $N$. californicum an der Westküste der USA, $N$. americanum in New Jersey, N. montanum in North Carolina (diese Art wird von anderen zu $N$. americanum gerechnet), $N$. ossifragum an westeuropäischen Küsten, $N$. reverchoni in Gebirgen auf Korsika, $N$. scardicum in Albanien und Nordgriechenland und $N$. balansae am Ostrand des Schwarzen Meeres.

Unser Narthecium führt mehrere deutsche Namen. Moorlilie bezieht sich auf den Wuchsort und die Ähnlichkeit der Blüten mit den Liliengewächsen. Ein anderer seltener Name ist Ährenlilie. Botanisch ist er falsch, denn der Blütenstand ist keine Ähre. Die alten Volksnamen Schoosterknief und Schusterkniep (Süssenguth 1939) beruhen auf der Gestalt der Blätter, die den Messern ähneln, die Schuster zum Zuschneiden des Leders benutzen. Der vermutlich gebräuchlichste Name Beinbrech oder drastischer ausgedrückt Knochenbruchgras (Kirchner I934, SchumaCHER I945) beschreibt, dass die Pflanze früher für Knochenbrüche beim Vieh verantwortlich gemacht wurde. Vermutlich beruht der Name auf dem kalkarmen Umfeld, in dem die Pflanze vorkommt, das dem Vieh wenig Kalk mit dem Futter lieferte und somit die Knochen der 
Tiere brüchig wurden. Vielleicht kommt der Name aber auch von den tiefgründigen, nassen Stellen in den Mooren, an denen das Vieh leicht einbrechen und sich dabei die Beine verletzen kann. Der Name wird zum ersten Mal 1667 von dem in dänischen Diensten stehenden Arzt Simon Paulli erwähnt, der den Brief eines norwegischen Befehlshabers auf Bergenshus (heute Bergen) veröffentlichte. In ihm wird die Futterpflanze "gramen norvegicum ossifragum" (norwegisches Beinbrechgras) genannt (Nielsen i979, Sauerhoff 2003). Dieser Volksglaube wird auch in dänischen, norwegischen und niederländischen Volksnamen der Pflanze wiedergegeben. Verwirrenderweise wird der Beinbrech manchmal als Beinheil bezeichnet (SCHUMACHER I945), nach BRÜNNER vor allem in Schleswig-Holstein und in der Lüneburger Heide. Angeblich soll er früher als Wundheilmittel (Verwechslung mit Beinwell?) und als innerliches Mittel gegen Harngries und Steinbeschwerden eingesetzt worden sein (BRÜNNER 1970). Für die Wirkung und die Anwesenheit besonderer Wirkstoffe gibt es aber keinerlei Hinweise. Die Bezeichnung Beinheil kann zu Verwechslungen mit der echten Heilpflanze Beinwell führen, einem Raublattgewächs (Boraginaceae), das dem Beinbrech weder ähnelt noch mit ihm näher verwandt ist und seit altersher für Wundheilungen benutzt wird.

Bei der wissenschaftlichen Namensgebung geht der Artbeiname ossifragum (Knochenbrecher) auf die oben erwähnten Zusammenhänge zurück, was aber von manchen Etymologen (GENAust 2005) wohl nicht ganz zutreffend als Legendenbildung zurückgewiesen wird. Auch die Herkunft des Gattungsnamens ist umstritten. Einige leiten Narthecium von narthex (griech. = Stab) ab, weil der kahle Stängel wie ein Stab aussieht. Als Narthex bezeichnen Botaniker aber eine bis $3 \mathrm{~m}$ hohe Pflanze, den Riesenfenchel (Ferula narthex), ein Doldengewächs, mit dem unsere Blume des Jahres

Abb. 1 (oben): Narthecium ossifragum, Habitus.

Abb. 2 (unten): Ausschnitt aus dem Blütenstand.
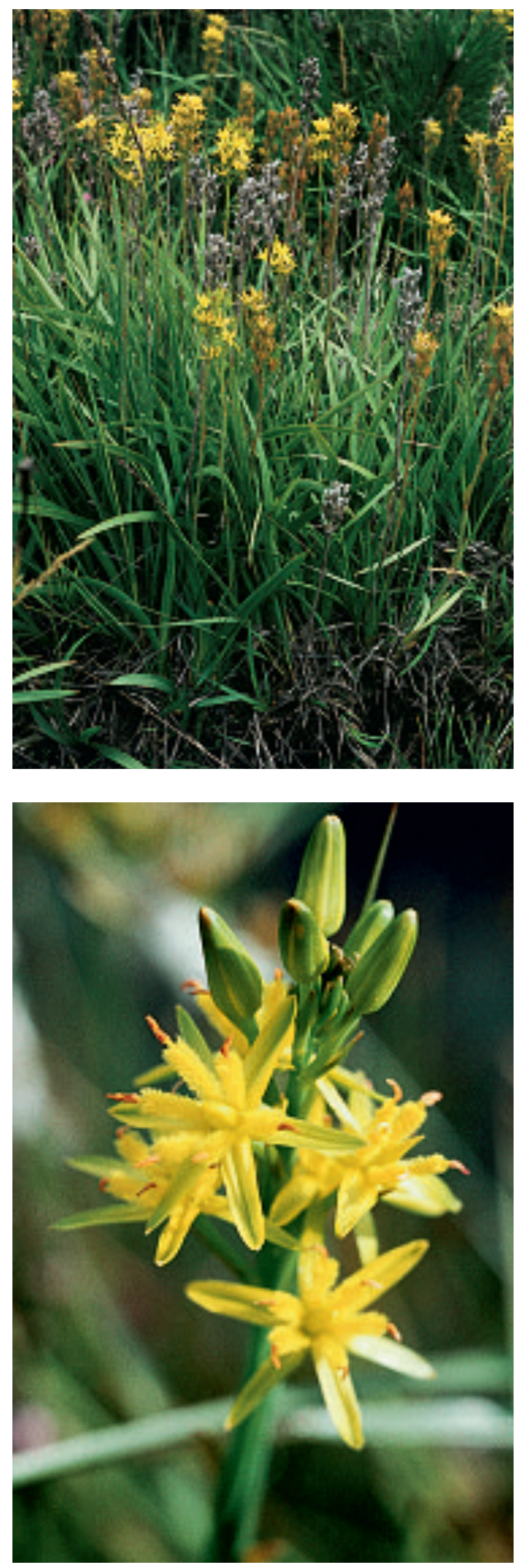


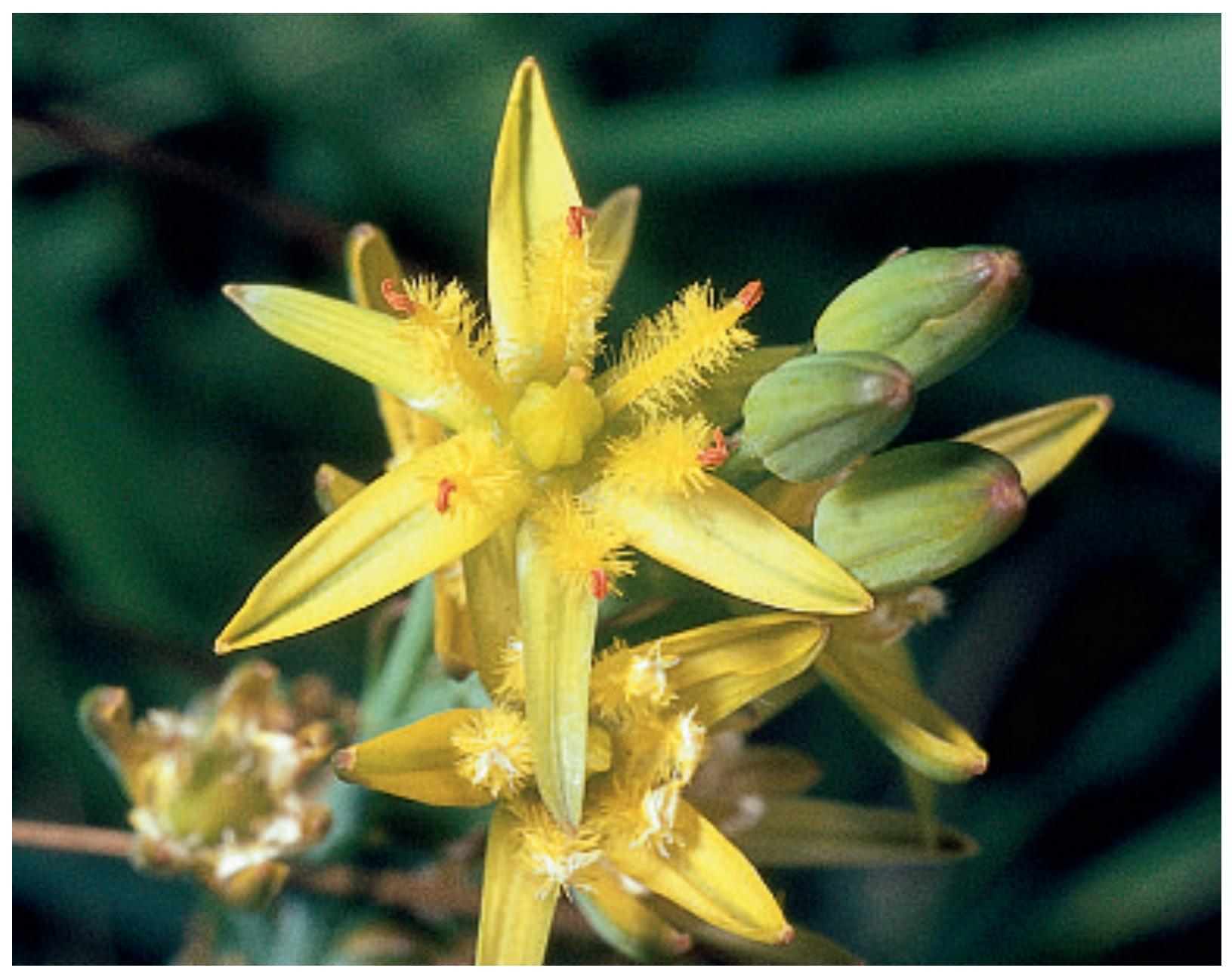

überhaupt keine Ähnlichkeit aufweist. GENAUST (2005) weist darauf hin, dass Narthecium ein Anagramm von Anthericum ist, ein Stilmittel, das der Erstbeschreiber Hudson durchaus benutzt hat. Da der Name Narthecium aber schon 20 Jahre vor Hudson von Moenring (1742) verwendet wurde, ist der Ursprung des Namens noch immer nicht eindeutig geklärt (DüLL \& KutZelnigg 20II).

\section{Vorkommen und Verbreitung}

Wie Eberle (1972) schreibt, ist der Beinbrech eine echt atlantische Pflanze, die häufig mit Gagelstrauch (Myrica gale), Glockenheide (Erica tetralix), Wassernabel (Hydrocotyle vulgaris), Mittlerem Sonnentau (Drosera intermedia) und Wollgräsern (Eriophorum) zusammen vorkommt. Beinbrech markiert die Grenze der subatlantischen Region, die auf den westlichen Teil Europas beschränkt ist mit relativ niedrigen Höchsttemperaturen, hohen Nie- derschlägen und sehr viel Wolken (SüsSENGUTH 1939). Beinbrech erträgt nicht länger anhaltende Lufttrockenheit, weshalb seine Wuchsgebiete sich im Wesentlichen auf die küstenbegleitenden Tiefländer beschränkt (Aichele 1996).

Sein Areal reicht vom Golf von Biskaya, über Großbritannien, Irland, das französische, belgische, niederländische, deutsche, dänische Küstengebiet und Südwestschweden entlang bis ins nördliche Norwegen (BRÜNNER 1970).

In seinem Verbreitungsgebiet wird Beinbrech als selten angegeben. An seinen Standorten bildet er kleine Bestände, die stellenweise dicht rasenartig sein können. (Ascherson \& GRAEBNER 1905). Hier wächst er in nassen Heidemooren und feuchten Heideplätzen der Geest, am besten in voller Sonne, in Glockenheide-Be-

Abb. 3: Blüte im Detail. 
ständen und zwischen Torfmoos. Er kommt aber auch in Gagel- und Weidengebüsch vor. Beinbrech ist schattenintolerant. Wenn das Gelände verbuscht, verschwindet er (SummerFIELD I974). Die Pflanze bevorzugt schlammigmoorige, stickstoffarme Böden oder feuchte Sande mit saurer Reaktion (Aichele 1996). Nach Weber (I995) sind es nasse, saure, wasserzügige Torfböden in Quell- und Zwischenmooren, aber auch kalkarmes Magergrünland. Wasser verträgt er, wenn es bis $10 \mathrm{~cm}$ unter der Erdoberfläche ansteht und in Bewegung ist. Die Böden zeigen einen $\mathrm{pH}-$ Wert zwischen 3,0 und 6,5 (Summerfield 1975). Während der Beinbrech im Flachland in dichten Gruppen kräftig wächst, blüht und fruchtet, bleibt er in größeren Höhen kleinwüchsig und oft steril (Schumacher 1945). Das Aufkommen des Beinbrechs wird begünstigt, wenn geeignete Flächen künstlich von Vegetation entblößt werden. Summerfield (1975) vermutet, dass das Beenden des so genannten Abplackens der Heide eine Ursache für den Rückgang des Beinbrechs ist.

\section{4. Äußeres Erscheinungsbild}

Beinbrech ist eine ausdauernde Pflanze. Sie entspringt einem kriechenden, dünnen 3-5 mm

Beinbrech kommt auch auf den Hebriden und den Färöer-Inseln vor. In Norwegen erreicht er eine Höhengrenze von $1100 \mathrm{~m}$. Normalerweise liegt seine Höhengrenze bei $600 \mathrm{~m}$ (Ascherson \& Graebner 1905). Während der Beinbrech in ganz Irland vorkommt, ist er in Großbritannien hauptsächlich in den nördlichen, westlichen und südwestlichen Landesteilen verbreitet, dagegen im zentralen und östlichen Bereich nur in isolierten Beständen. Auch früher war er auf die "green sand heath" im westlichen Norfolk beschränkt (Summerfield 1974). Die früher erwähnten Vorkommen im Baltikum sind fraglich, die in Tschechien Falschmeldungen (Schumacher i945). Die Funde aus dicken, sich verzweigenden Wurzelstock (Rhizom). Die Wurzeln entstammen leicht geschwollenen Knotenbereichen. Das Rhizom liegt oberflächennah in meist 2-10 (seltener bis 25) $\mathrm{cm}$ Tiefe. Das Rhizom ist mit kurzen scheidigen Niederblättern besetzt und endet in einem kleinen, unterirdischen Faserschopf aus abgestorbenen Niederblättern. Ein Mykorrhiza-Pilz befindet sich in den Wurzeln und im Rhizom.

Jeder Blütentrieb benötigt mehrere (2-7) Jahre zur Erstarkung. Das Rhizom setzt sich daher aus mehreren monopodial verketteten Jahrestrieben zusammen (Acherson \& Graebner I905). Der jährliche Zuwachs beträgt $2-5 \mathrm{~cm}$ (Kirchner \& Loew I934). So entsteht jährlich ein Laubtrieb, der als Knospe im voraufgegangenen Herbst in der Achsel des obersten Blattes angelegt wurde. Im Winter ist die $1 \mathrm{~cm}$ große Knospe durch die faserigen, vertrockneten Niederblattscheidenreste umhüllt und geschützt. Die Seitentriebe bilden sich aus den Achselknospen an anderen Blättern. An günstigen Stellen kommt es zur reichlichen Bildung von Trieben nach allen Seiten (Schumacher I945). Im Blühjahr werden keine Seitensprosse gebildet. Die Seitensprosse gelangen nach mehreren Jahren zur Blüte.

Korsika stellt man heute zu einer anderen Art (Narthecium reverchoni).

In Deutschland kommt der Beinbrech in einem Gebiet vom Hohen Venn über Hannover, Hamburg, Ostfriesland bis in die Geestgebiete im Westen von Schleswig-Holstein vor. Er fehlt allgemein in der Marsch (BRÜNNER 1970), überschreitet die Elbe nur in ihrem unteren Lauf und fehlt völlig in Mecklenburg-Vorpommern und Brandenburg. Sein östlichster Fundort ist Ilmenau in der Lüneburger Heide (Eberle I972). Am Rand der Mittelgebirge Nordrhein-Westfalens finden sich nur ganz vereinzelte Vorkommen (Ascherson \&GraebNer I905, Aichele 1996). 


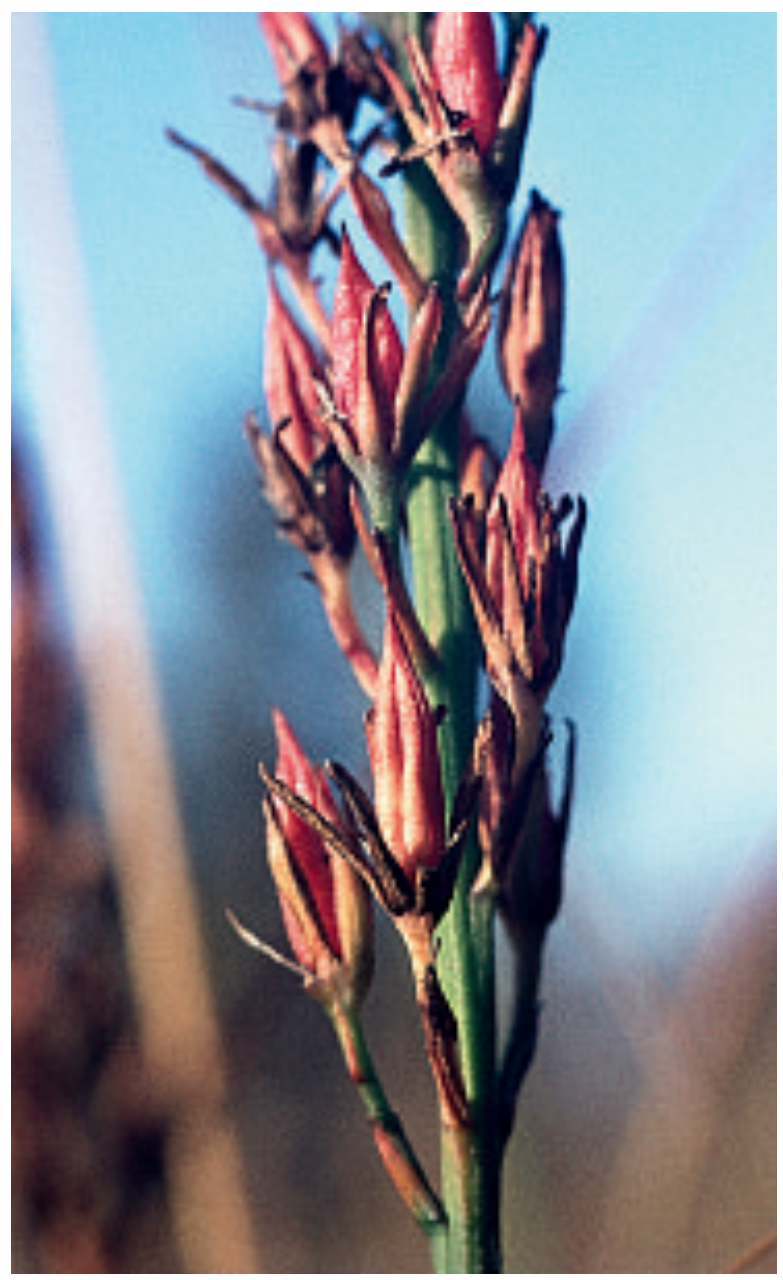

Die Laubsprosse entwickeln 6-8 streng zweizeilig, senkrecht gestellte Blätter mit Längsfaltung. Sie sitzen mit ihrer unterwärts offenen, schmal weiß berandeten Scheide der Sprossachse quer auf. Es sind linealische, schwertförmige, reitende und kahle Blätter. Sie sind im oberen Drittel scharf zugespitzt und häufig vom Grund bis zur Spitze gekrümmt. Die Blätter sind schwach xeromorph, was in diesem Fall nicht mit Wassermangel, sondern mit Nahrungsmangel zusammenhängt, wie es auch für andere Moorpflanzen bekannt ist (Kirchner \& Loew 1934). Die Blätter sind 5-30 cm lang und etwa 2-5 mm breit (Weber 1995) Die Stängelblätter am Blütentrieb sind nur 2-3 cm lang, die obersten ähneln den Blütentragblättern. Ein Unterscheidungsmerkmal zu der vegetativ sehr ähnlich aussehenden Torf- oder Simsenlilie (Tofieldia) sind die sehr kurzen Scheidenblätter am Stängelgrund (Kirchner \& Loew 1934).
Der Blütenstängel ist wenig beblättert. Er wird 10-30, maximal $40 \mathrm{~cm}$ hoch. Er steht starr aufrecht, ist klebrig, deutlich gerieft und hat eine rötliche Farbe. Dies ist besonders auffällig bei den bis zum nächsten Jahr stehen bleibenden Stängeln.. Der Blütenduft erinnert an Nelken (SüsSENGUTH I939).

\section{Blüte und Frucht}

Der endständige Blütenstand ist eine Traube, denn die Einzelblüten können länger als $1 \mathrm{~cm}$ gestielt sein (AiCHELE 1996). Er wird $5-8 \mathrm{~cm}$ lang. Nach Aichele sind es selten mehr als 10 Blüten, nach anderen Quellen sogar bis 20. Dem Blütenstand fehlt meist eine Gipfelblüte. Die Blütezeit liegt in Deutschland im Juli und August.

Die Blüte steht in der Achsel eines linealischlanzettlichen Tragblattes. Sie umfasst sechs linealische, radspeichenförmig abstehende Blütenblättern (KRAUSe 1930). die 6-9 mm lang, innen gelb und außen grün mit dunkelgrünem Mittelstreifen sind. Die Blütenblätter fallen nicht $a b$, sondern falten sich nach der Blütezeit zusammen. Vor jedem Blütenblatt steht ein Staubgefäß mit ziegelroten, gedrehten, nach innen aufspringenden Staubbeuteln. Die Farbe kontrastiert mit der dichten wolligen Behaarung des Staubfadens. Über die Funktion der Staubfadenhaare gibt es bis heute nur Vermutungen (Schumacher 1945). Die Haare werden für eine Pollenfalle gehalten, die Regentropfen erlauben, den gesammelten Pollen auf die Narbe schwimmen zu lassen. Summerfield (1974) berichtet über Beinbrech-Pflanzen auf den Färöer-Inseln, dass Regen zur Bestäubung beiträgt. In den vom Regenwasser gefüllten Blüten wird der Pollen auf der Wasseroberfläche zur Narbe transportiert.

Selbstbestäubung ist beim Beinbrech erschwert, da die Staubbeutel $3 \mathrm{~mm}$ von den Narben entfernt stehen. Nach KIRCHner et al. die-

Abb. 4: Fruchtendes Exemplar.

Abb. 5 (Seite 131): Narthecium americanum. Historische Illustration aus Curtis's Botanical Magazine, 1813. 
nen die Staubfadenhaare möglicherweise dazu, dass sich die blütenbesuchenden Insekten daran festhalten können. Da es sich um größere Insekten wie Hummeln, Honigbienen, aber auch kleinere Wildbienen und Fliegen handelt, ist nicht geklärt, wie weit das mechanisch möglich ist. Sie könnten sich dabei natürlich mit dem anhaftenden Pollen bepudern. Nach SchumaCHER soll Beinbrech meist vom Wind bestäubt werden. Dann könnten die Haare eine Hilfseinrichtung für das Pollensammeln bei Windstille sein, wie man das auch bei Grasblüten kennt (Düll \& Kutzelnigg 20II). Der oberständige dreikammerige Fruchtknoten geht in einen Griffel über, der eine dreilappige Narbe bildet (Ascherson \& Graebner 1905). Die Blüte bildet keinen Nektar, hat aber einen Duft, der an Waldhyazinthen (Platanthera) erinnert. Nach dem Verblühen werden Stängel, Blütenblätter und Frucht dunkelorange (SummerFIELD 1975) und bilden einen auffallenden farbigen Akzent in den Mooren.

Die Frucht ist eine aufrecht stehende, ovale, schlanke, zugespitzte Kapsel von 12 mm Länge. Die Samen werden vom Wind ausgebreitet. Sie sind bis 1,2 $\mathrm{mm}$ lang und haben an beiden Enden fadenförmige, bis 3,5 mm lange Anhängsel. Die Samen benötigen für den Keimungsbeginn keine Kälteeinwirkung. In den ersten beiden Jahren bilden die Jungpflanzen unter künstlichen Bedingungen kein Rhizom aus. Die Keimfähigkeit der Samen bleibt bis zum Spätsommer des folgenden Jahres erhalten (Summerfield 1975). Eberle (1975) berichtet von einem Aussaatversuch am natürlichen Standort, bei dem es nach 9 Jahren zur ersten Blüte kam.

\section{Literatur}

Aichele, D., Schwegler, H. W. 1996: Die Blütenpflanzen Mitteleuropas. Bd. 5. -Stuttgart.

Ascherson, P. \& Graebner, P. I905-07: Synopsis der mitteleuropäischen Flora, Bd.13. - Leipzig.

BRÜNner, G. 1970: Beinbrech und Beinheil. - Kosmos 66: 415-417.

BRUmmit, R. K. I992: Vascular plant families and genera. - Kew.

Düll, R. \& Kutzelnigg, H. 20i i: Taschenlexikon der Pflanzen Deutschlands und angrenzender Länder. 7. Aufl. - Wiebelsheim.

Eberle, G. I972: Lilien und Lilienverwandte in den Floren Mittel- und Südeuropas. - Frankfurt.

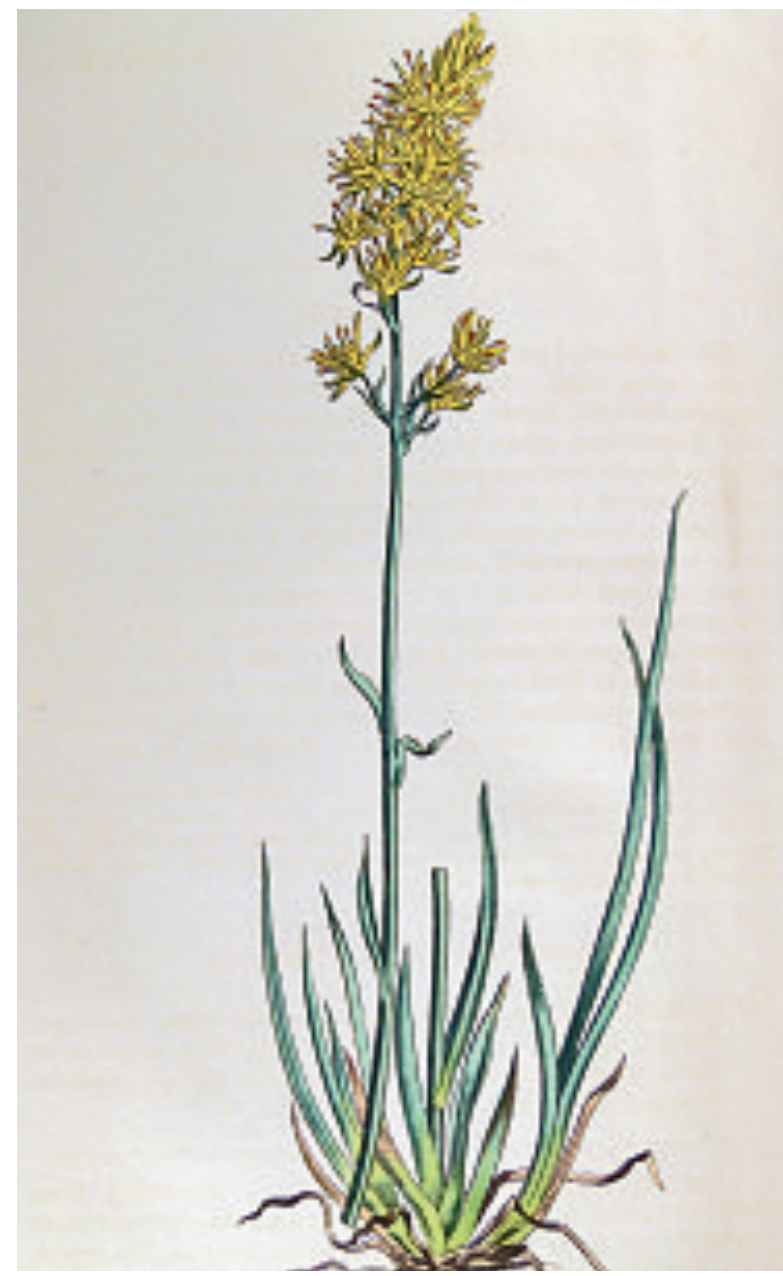

Genaust, H. 2005: Etymologisches Wörterbuch der botanischen Pflanzennamen. 3. Aufl. - Hamburg. Haeupler, H. \& Muer,T. 2007: Bildatlas der Farn- und Blütenpflanzen Deutschlands. 2. Aufl. - Stuttgart. Kirchner, O., Loew, E. \& Schröter, C. I934: Lebensgeschichte der Blütenpflanzen Mitteleuropas. Bd. I Abt. 3. - Stuttgart.

Krause, K. I930: Liliaceae. In : Engler, A. \& Prantl, K.: Die natürlichen Pflanzenfamilien. Bd. 15 a. 2. Aufl. - Leipzig.

Mabberley, D. J. 2008: Mabberley's plant book. 3. Aufl. - Cambridge.

Nielsen, H. I979: Giftpflanzen. - Stuttgart.

Sauerhof, F. 2003: Etymologisches Wörterbuch der Pflanzennamen. - Stuttgart.

Schumacher, A. 1945: Die Moorlilien (Narthecium) - Arten Europas. - Archiv Hydrobiol. 41: 112-195. Süssenguth, K. I939: Liliaceae. In: Hegi, G. (Hrsg.): Illustrierte Flora von Mitteleuropa. Bd. II. 2. Aufl. - München.

Summerfield, R. J. I974: Biological flora of the British Isles. Narthecium ossifragum. - J. Ecol. 62: 325-339.

Tamura, M. N. I998: Nartheciaceae. In: Kubitzki, K.: The families and genera of vascular plants. Bd. 3. - Berlin. Weber, H. 1995: Flora von Südwest-Niedersachsen und des benachbarten Westfalens. - Osnabrück. 